\title{
Usefulness of contrast-enhanced ultrasound using perfluorobutane- containing microbubbles as a planning for percutaneous biopsies of focal hepatic lesions: a prospective feasibility study
}

\author{
Sang Min Lee1, Jung Hoon Kim²,3, Hyun Kyung Yang², Hyo-Jin Kang², Joon Koo Han ${ }^{2,3}$
}

${ }^{1}$ Department of Radiology, Hallym University Sacred Heart Hospital, Gyeonggi-do, ${ }^{2}$ Department of Radiology, Seoul National University Hospital, Seoul, ${ }^{3}$ Institute of Radiation Medicine, Seoul National University College of Medicine, Seoul, Korea

\begin{abstract}
Aims: To determine whether contrast-enhanced US using perfluorobutane-containing microbubbles (SEUS) would be helpful for planning a hepatic biopsy. Material and methods: This prospective study included 40 patients who planned to undergo hepatic biopsy for focal hepatic lesions. All patients underwent B-mode US followed by SEUS. The radiologist evaluated the number of detected lesions, presence of necrosis, conspicuity of target lesion and technical feasibility using 4-point scale. Technical failure and occurrence of change of the target were also assessed. Computer tomography (CT) or magnetic resonance (MR) images were the reference techniques. Results: The mean number of lesions detected on CT and MR images was $6.5 \pm 8.4$. In $20(50 \%)$ of 40 patients, more focal lesions were detected on SEUS. Targeted lesion was changed in six patients $(15 \%)$ on SEUS. Mean number of detected lesions on SEUS was significantly higher comparing with B-US (5.1 \pm 6.2 vs. $2.8 \pm 3.8, \mathrm{p}<0.001)$. Conspicuity of the targeted lesion was improved in $67.5 \%$ (27 of 40$)$ on SEUS and significantly more visualized than B-US $(3.6 \pm 0.8$ vs. $2.8 \pm 0.9, \mathrm{p}<0.001)$. In 7 more patients the necrosis within the lesion was visualized $(17.5 \%)$ using SEUS. The technical feasibility on SEUS was significantly higher than B-US $(2.3 \pm 1.0 \mathrm{vs.} 3.3 \pm 0.9$, p $<0.001)$. Technical failure was observed in only one patient (2.5\%). Conclusions: SEUS is a helpful technique for planning the hepatic biopsy in terms of detection, improving lesion conspicuity, tumor viable portion assessment and consequently higher operator confidence, compared with B-US.
\end{abstract}

Keywords: liver; biopsy; neoplasms; ultrasonography; perfluorobutane-containing microbubbles

\section{Introduction}

Histopathologic diagnosis is crucial for the management strategy and the optimal treatment planning in patients with focal hepatic lesions [1]. Ultrasonography (US) allows operators to simultaneously observe focal hepatic lesions and to perform percutaneous biopsy of

Received 06.10.2018 Accepted 16.01.2019

Med Ultrason

2019, Vol. 21, No 2, 109-116

Corresponding author: Jung Hoon Kim, M.D.

Department of Radiology, Seoul National

University Hospital, 101 Daehangno,

Jongno-gu, Seoul, 110-744, Republic of Korea

Phone: +82-2-2072-1969

Fax: +82-2-743-6385

E-mail: jhkim2008@gmail.com a lesion in real time. As a result, US-guided biopsy of focal hepatic lesions may be easier and safer to perform than ever before. Nevertheless, US-guided percutaneous biopsy is still an invasive procedure which may cause various complications, including life-threatening bleeding. Therefore, the safety and success rate of percutaneous biopsy of focal hepatic lesions is an important issue and it is essential to obtain accurate and appropriate tissue without complications.

Even though the detection and conspicuity of focal hepatic lesions on US is a key factor determining the success or failure of US-guided percutaneous biopsy [2], not all focal hepatic lesions are easily visible on conventional B-mode US [3]. To overcome the visibility problem of Bmode US, several techniques, including fusion imaging and contrast-enhanced US (CEUS) have been developed [2,4-6]. CEUS utilizes the nonlinear responses from the 
ultrasound contrast microbubbles to enhance tissue [7]. Focal hepatic lesions can be delineated by differences of contrast flow between the lesions and the surrounding hepatic parenchyma [8]. With this mechanism, CEUS increases the lesion-to-liver contrast ratios, and thus making focal hepatic lesions more conspicuous.

There is now a perfluorobutane-containing microbubble contrast agent (Sonazoid, GE Healthcare, Oslo, Norway) consisting of perfluorobutane with a phospholipid shell [9]. Unlike previously introduced ultrasound agents $[10,11]$, Sonazoid allows parenchyma-specific liver imaging, termed Kupffer-phase imaging, as it is taken up by Kupffer cells in the reticuloendothelial system of the liver $[12,13]$. Malignant lesions that have few or no Kupffer cells can be well-delineated as contrast defects during the Kupffer-phase beginning 10 minutes after administration, while surrounding normal hepatic parenchyma containing normal Kupffer cells appears well-enhanced [14]. Kupffer phase imaging can then be performed for even more than several hours and can provide stable images for longer time and overcome the narrow time window of previously used ultrasound contrast [15].

Several studies regarding the usefulness of CEUSguided biopsy $[4,8,16-18]$ have been published. However, only a few studies have been reported using perfluorobutane-containing microbubble contrast agent for percutaneous biopsy or radiofrequency ablation (RFA) of focal liver lesions $[5,6,19]$. Furthermore, there is no published study to assess whether perfluorobutane-containing, microbubbles-enhanced US (SEUS) achieves a change in the biopsy target and helps to determine the appropriate target when planning a biopsy of focal hepatic lesions.

Therefore, the purpose of this study was to determine whether SEUS would be helpful for planning the percutaneous biopsies for focal hepatic lesions and focused on improving lesion detection, tumor viable portion assessment and consequently higher operator confidence, compared with B-mode US.

\section{Materials and methods}

This prospective study was approved by our Institutional Review Board, and written informed consent was obtained from all patients before SEUS and the biopsy procedure.

\section{Patients}

From September 2016 to February 2017, a total of 332 patients were referred for percutaneous liver biopsy for focal hepatic lesions. The flow diagram of the patient enrollment is summarized in figure 1. Finally, a total of 40 patients (mean age, 57.2 years \pm 11.2 [standard deviation];

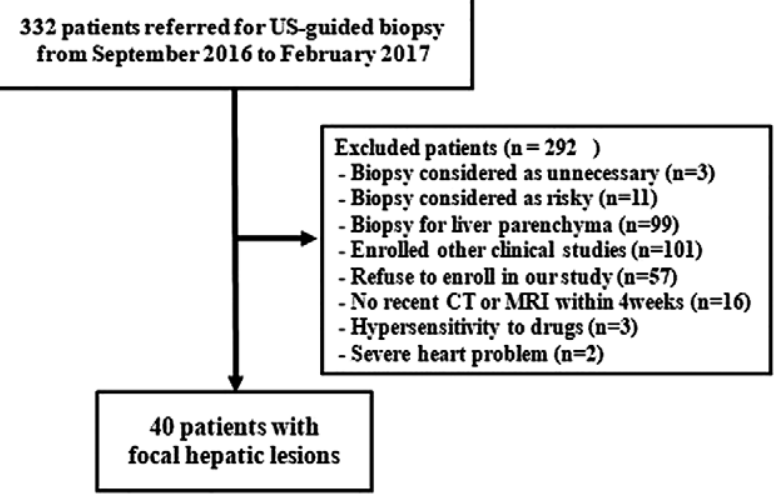

Fig 1. Flow diagram of patient population

age range, 21-78 years) were prospectively enrolled in this study. None of the patients had any contraindication for liver biopsy, such as a bleeding tendency. Ten of the 40 patients had a history of chronic liver disease. All the patients included in this study underwent computer tomography (CT) or magnetic resonance (MR) images before the biopsy and had focal hepatic lesions seen on CT ( 28 cases) or MR images (12 cases). The mean time interval between CT/MRI and the biopsy was $14.5 \pm 16.1$ days. The baseline patient demographics are shown in Table I.

Table I. Summary of baseline patient demographics

\begin{tabular}{ll}
\hline Characteristic & Value \\
\hline Age, Mean years \pm SD & $57.2 \pm 11.2(21-78)$ \\
Sex, Male:Female & $32: 8$ \\
Past history & \\
$\quad$ Chronic liver disease & 10 \\
$\quad$ Hepatitis B virus & 7 \\
Alcohol & 1 \\
Cirrhosis of unknown etiology & 2 \\
History of malignancy & 18 \\
Hepatocellular carcinoma & 3 \\
Lung cancer & 3 \\
Gastric cancer & 2 \\
Colon cancer & 1 \\
AOV cancer & 1 \\
CBD cancer & 1 \\
Breast cancer & 1 \\
GIST & 2 \\
Renal cell carcinoma & 1 \\
Nasopharyngeal cancer & 1 \\
Malignant melanoma & 1 \\
Neuroendocrine tumor & 1 \\
Hepatocellular carcinoma & 3 \\
Reference images, CT:MR & $28: 12$ \\
\hline
\end{tabular}

AOV - Ampulla of Vater; CBD - Common Bile Duct; GIST - Gastrointestinal Stromal Tumor; CT - Computer Tomography; MR Magnetic Resonance 


\section{US techniques}

US was performed by one of the two board-certified abdominal radiologists (S.M.L. and H.K.Y.) with five to nine years' experience in ultrasound imaging. The examiners were aware of each patient's past medical history and the purpose of this study. A conventional US unit (RS80A, Samsung Medison, Seoul, Korea) with a 1-7 $\mathrm{MHz}$ convex probe with a center frequency of $1.3 \mathrm{MHz}$ (CA1-7A, Samsung Medison, Seoul, Korea) was used. The US parameters were as follows: mechanical index of 1.3-1.4, gain of $40-70 \%$, dynamic range of 50 and a frame rate of 26 to 32 pictures/s. Before the US examination, the previous $\mathrm{CT}$ or MR images were reviewed and regarded as reference standards. For proper B-mode US examination for the detection of target lesions, parameters including the gain and focal zone were optimized by the operator. Different positions including the intercostal or subcostal approach were applied.

\section{Contrast-enhanced US techniques}

Subsequently, SEUS was performed using the same US system by the same operator. The perfluorobutanecontaining microbubble contrast agent (Sonazoid, GE Healthcare, Oslo, Norway) was reconstituted with $2 \mathrm{~mL}$ of sterile water for injection. One vial of Sonazoid was divided into two doses, each of $1 \mathrm{ml}$. The first dose was used for pre-biopsy planning and the second dose was used for the targeted biopsy by a manual bolus injection followed by a flush with $10 \mathrm{~mL}$ of normal saline via the ante-cubital venous line in each administration. The first dose was used for the detection of focal lesions in the entire liver and the second dose was injected for the target lesion with a fixed single plane. During the second injection the arterial enhancement of the hepatic focal lesion was evaluated. The mechanical index was automatically lowered to 0.198 and the level was adjusted depending on the location and depth of the lesion. Images were obtained at a transmission frequency of $2.4 \mathrm{MHz}$, a frame rate of $9 \mathrm{~Hz}$, a dynamic range of 50, a gain of 63 , and a depth of $12-15 \mathrm{~cm}$. SEUS was performed using the asymmetric pulse modulation image mode (PEN2; Samsung Medison, Korea). During the first dose, the liver was continuously scanned during the vascular phase (approximately three minutes) under normal calm breathing and was intermittently scanned until the Kupffer phase (approximately 10-15 minutes). During the first dose the target lesion and the safe route for USguided biopsy was determined. The images were obtained with both single shot and cine loops and were sent to the Picture Archiving and Communication System (PACS) and were subsequently analyzed by the same operator.

\section{Biopsy procedure}

US-guided biopsy was performed by the same radiologists (S.M.L. and H.K.Y.) with experience of $>200$ cas- es requiring US-guided biopsy. For the biopsy procedure, the same US system was used for US guidance. The operator chose the target lesion from all the detected lesions and determined the biopsy route considering the SEUS findings in order to avoid vessel injury and achieve adequate tissue sampling. The percutaneous liver biopsy was performed with an 18-gauge, automated, side-cutting biopsy gun (Acecut; TSK Laboratory, Tochigi, Japan) using the free-handle technique. We obtained at least two biopsy specimens and repeated sampling was performed if visual inspection of a specimen was doubtful of the technical success. The number of samplings was determined based on the operator's judgement.

\section{On-site image analysis}

The operator conducting the biopsy evaluated the number of lesions, the conspicuity of the target lesion, the presence of necrosis within the target lesion, and the technical feasibility of biopsy on B-mode US and CEUS. The operator evaluated also the number of the focal lesions and the location of the target lesion on the previous CT or MR images. Necrosis within the target lesion was defined as an anechoic area seen on B-mode US and a non-enhancing area during the vascular phase of the SEUS. The conspicuity of the target lesion was scored on a 4-point scale, with a score of 1 indicating definitively non-visualized, score 2 slightly visible and $<50 \%$ of the lesion with a well-defined margin, score 3 fairly visible and $>50 \%$ of the lesion with a well-defined margin, and score 4 definitively visible and distinguishable from the surrounding liver parenchyma and $>90 \%$ of the lesion being visible [20]. The technical feasibility was evaluated depending on the operator's confidence based on a combination of lesion visibility and safety routes, which were graded as: score 1 indicating not feasible due to an invisible lesion or a poorly safe access route; score 2 indicating equivocally feasible due to a partially visible lesion with a fairly safe route; score 3 indicating fairly feasible due to fair lesion conspicuity and a fairly safe access route and score 4 indicating definitely feasible due to good lesion visibility and a good safe access route [21]. Technical failure was considered when SEUS-guided biopsy could not be performed due to non-visualization of the target lesion twice on SEUS or non-visualization of a safe access route. The cases in which the biopsy result was inadequate and needed a rebiopsy were also regarded as a technical failure. Immediate complications were assessed on the spot and delayed complications $(<24$ hours) were evaluated through the electric medical records. When the pathologic result was positive for neoplasm, the pathologic diagnosis through biopsy was considered as the final diagnosis. Otherwise, the final diagnosis was established based on the patho- 
logical examination of the biopsy specimen as well as on the clinical and radiology follow-up.

\section{Statistical analysis}

Statistical analyses were performed using commercially available software programs (SPSS, version 23; SPSS, IBM, Armonk, NY, USA; or MedCalc, version 16, MedCalcSoftware, Mariakerke, Belgium). In order to compare the number of detected lesions, the conspicuity of the target lesion, and the subjective technical feasibility on B-mode US and SEUS, a paired T-test was used. The detection rate of necrosis within the target lesion was compared between B-mode US and SEUS using the McNemar test. The chi-square test was used to determine whether detection of necrosis within the lesion was or was not associated with change of the target lesion and the target portion. To determine whether the number of detected lesions on B-mode US and SEUS differed from those of the reference image, a pair-wise comparison with the paired t-test was used. $\mathrm{p}<0.05$ was considered to indicate statistical significance. All numerical data were expressed as mean values \pm standard deviations (SD).

\section{Results}

Technical failure on SEUS as well as on B-mode US was observed in one patient whose target lesion was located in the subdiaphragmatic area and was non-visualized. In that case, percutaneous biopsy was appropriately performed with the addition of fusion imaging. No patient showed an inappropriate diagnosis and required re-biopsy. Thirty-three of the 40 patients $(82.5 \%)$ had a definitive pathologic diagnosis through biopsy: 28 malignant neoplasms (hepatocelluar carcinoma [ $\mathrm{n}=8]$, intrahepatic cholangiocarcinoma $[\mathrm{n}=3]$ and hepatic metastasis $[\mathrm{n}=17]$ ) and 5 benign neoplasms (focal nodular hyperplasia [ $\mathrm{n}=3$ ], cavernous hemangioma [ $\mathrm{n}=1]$ and peliosis hepatitis $[\mathrm{n}=1])$. The remaining seven patients had no definitive pathologic diagnosis through biopsy; however, their pathology results were helpful for making the final

Table II. Comparison of the results of B-mode ultrasonography and SEUS as a planning for percutaneous biopsies of focal hepatic lesions

\begin{tabular}{llll}
\hline & B-mode US & SEUS & p value \\
\hline $\begin{array}{l}\text { Number of detected } \\
\text { lesions }\end{array}$ & $2.8 \pm 3.8$ & $5.1 \pm 6.2$ & $<0.001$ \\
$\begin{array}{l}\text { Conspicuity of the } \\
\text { target lesion }\end{array}$ & $2.8 \pm 0.9$ & $3.6 \pm 0.8$ & $<0.001$ \\
$\begin{array}{l}\text { Necrosis within the } \\
\text { target lesion }\end{array}$ & 4 & 11 & $\mathrm{p}=0.016$ \\
Technical feasibility & $2.3 \pm 1.0$ & $3.3 \pm 0.9$ & $<0.001$ \\
\hline
\end{tabular}

The results are expressed as number or mean \pm standard deviation diagnosis (focal steatosis [ $\mathrm{n}=2]$, inflammation $[\mathrm{n}=2]$, and neutrophilic cholangitis $[\mathrm{n}=1]$ ) after clinical and imaging follow-up for 8 to 30 weeks. No major, procedure-related complications requiring additional treatment or hospitalization occurred. One patient showed a mild hypersensitivity reaction immediately after contrast injection and recovered after a short amount of time. The mean size of the target lesion measured on the CT and MR images was $3.2 \pm 2.2 \mathrm{~cm}$. The target lesions were distributed in segment $2(\mathrm{n}=1)$, segment $3(\mathrm{n}=2)$, segment $4(\mathrm{n}=6)$, segment $5(\mathrm{n}=15)$, segment $6(\mathrm{n}=3)$, segment $7(\mathrm{n}=4)$ and segment $8(\mathrm{n}=9)$.

The mean number of detected lesions on $\mathrm{CT}$ and MR images was $6.5 \pm 8.4$. In $20(50 \%)$ of the 40 patients, more focal lesions were detected on SEUS than on B-mode US. The results of the B-mode US and SEUS are detailed in Table II. While the number of detected lesions on B-mode US was significantly lower than that on the CT and MR images $(\mathrm{p}<0.001)$, the number on SEUS detected lesions was comparable to that on the CT and MR images $(p=0.034)$. In addition, in 5 out 40 patients $(12.5 \%)$ SEUS showed more lesions than the CT/MR images.

The conspicuity of target lesions was improved in $67.5 \%$ (27 of 40 patients) using SEUS. Although more than one lesion was visible in 35 patients, focal hepatic lesions detected on CT/MR were not visualized in 5 of the 40 patients on B-mode US. In 4 of these 5 patients, the lesions were visualized on SEUS (fig 2) and the US guided biopsy was performed using the dual split screen mode with B-mode and Kupffer phase image of SEUS.

The conspicuity of the target lesion on SEUS was significantly improved $(p<0.001)$. The detection rate of necrosis within a lesion differed significantly between B-mode US and SEUS ( $\mathrm{p}=0.016$; fig 3). Regarding the procedure safety and proper tissue sampling, the target lesion determined on B-mode US was changed in six of the 40 patients (15\%) on SEUS and the target portion expected on B-mode US was also changed in nine of the 40 patients $(22.5 \%)$ on SEUS. The change of the target portion was significantly associated with the additional detection of necrosis within the lesion on SEUS $(p<0.001)$. In 7 patients in whom the necrosis within the lesion was more visualized on SEUS, the target portion was changed after SEUS in 5 patients and the target lesion was changed in 2 patients. The technical feasibility on SEUS was significantly improved $(p<0.001)$ in 32 $(80.0 \%)$ of the 40 lesions.

\section{Discussions}

Our results demonstrate that SEUS increased lesion detection, improved lesion conspicuity and improved vis- 


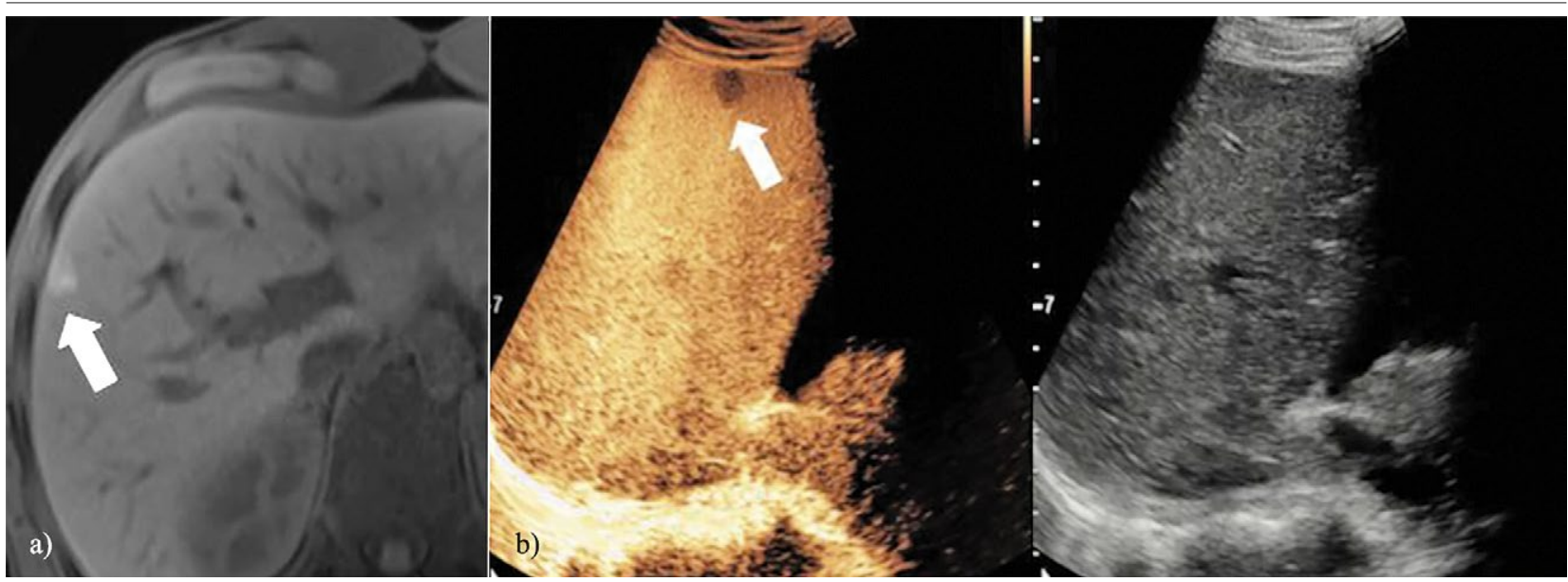

Fig 2. Hepatic metastasis from melanoma in a 37-year-old man: a) pre-contrast T1WI shows a hyperintense lesion in segment 8; b) Split-screen of SEUS during Kupffer phase (left) and B-mode US (right) show a focal hypoechoic lesion (arrow) on SEUS. However, this lesion was invisible on B-mode US.

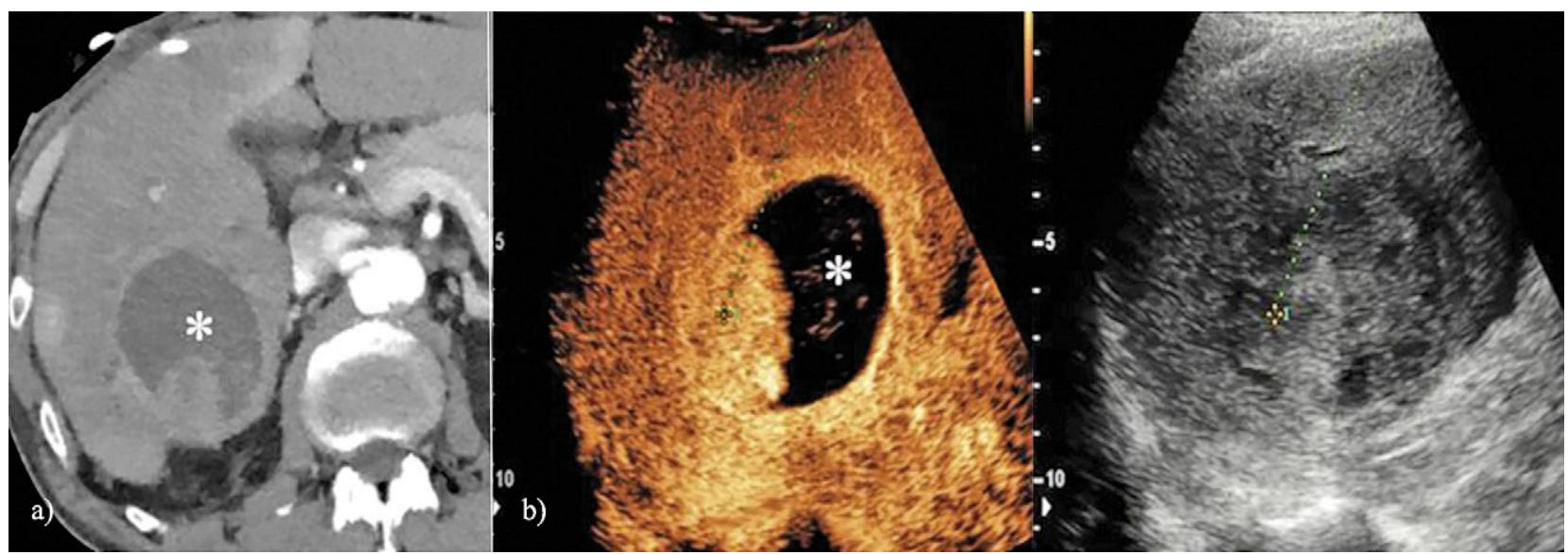

Fig 3. Hepatocellular carcinoma in a 60-year-old man: a) contrast enhanced CT during arterial phase shows an enhancing mass with necrosis (asterisk) in right lobe of the liver; b) split-screen of SEUS during vascular phase (left) and B-mode US (right) show an enhancing mass with necrosis (asterisk). However, B-mode US could not delineate necrotic portion within the mass. The percutaneous biopsy for non-necrotic portion could be performed under the guidance of SEUS and pathologic examination revealed hepatocellular carcinoma.

ualization of necrosis within a lesion, compared with Bmode US. Therefore, the target lesion determined by the operator on B-mode US was changed in 6 out 40 patients $(15 \%)$ on SEUS and the target portion expected on Bmode US was also changed in 9 out 40 patients $(22.5 \%)$ on SEUS. In addition, the technical feasibility improved while performing B-mode US followed by SEUS for biopsy planning, and thus yielding a high technical success rate of $97.5 \%(39 / 40)$.

According to the previous study comparing B-mode US and SEUS including the vascular phase and Kupffer phase, the lesion conspicuity score was significantly better on Kupffer-phase $(4.36 \pm 1.18)$ than on vascular phase (3.97 \pm 1.41$)$ and B-mode US (3.29 \pm 1.25$)$ [5]. Kang et al reported that the lesion conspicuity in 15 of 16 lesions (93.8\%) was increased after adding SEUS, as compared to that on fusion imaging [6]. In our study, the conspicuity of the target lesion on SEUS was significantly improved in $67.5 \%$ lesions, results that are consistent with the previous published studies $[5,6]$. The number of detected lesions in SEUS was slightly lower than that of CT/MR images, but in five patients (12.5\%) SEUS showed more lesions than $\mathrm{CT} / \mathrm{MR}$ images, i.e. the reference images. Mishima et al found also that SEUS has better detection for small hepatic metastasis compared with CT [22].

A previous, large-scale study regarding US-guided biopsy of focal hepatic lesions reported that all biopsy specimens showing insufficiency for diagnosis were 
caused by tissue necrosis with poorly preserved cell structure [23]. According to Bang et al, CEUS using Levovist (Schering AG, Berlin, Germany) identified the viable portion in necrotic masses, consequently obtaining adequate specimens [24]. Yoon et al reported that CEUS using SonoVue (Braccon, Milan, Italy) visualized the non-necrotic portion within a necrotic hepatic lesion in one of 44 cases included in the study and therefore successful biopsy was possible [4]. We also found that SEUS had a higher detection rate of necrosis within the lesion than B-mode US and this observation was significantly associated with change of the target portion. To the best of our knowledge, this is the first study which statistically proved that SEUS improved the detection of necrosis within a lesion allowing the target portion to be properly selected.

In our study, technical failure was observed in one patient $(1 / 40,2.5 \%)$ whose target lesion was located in the subdiaphragmatic area and was non-visualized not only on B-mode US but also on SEUS. The subdiaphragmatic location was known as a blind spot on both B-mode US and CEUS $[25,26]$. In this case with the aid of fusion imaging, percutaneous biopsy was properly performed. Fusion imaging has been used as a guidance tool for biopsy and radiofrequency ablation of focal hepatic lesions, the technique being able to increase the lesion conspicuity and technical feasibility, resulting in a high technical success rate $[2,21,27]$. However, Lee et al reported that $13.1 \%(13 / 99)$ of small hepatocellular carcinomas (HCCs) between $1 \mathrm{~cm}$ and $2 \mathrm{~cm}$ in size were still invisible on planning US for RFA with the addition of fusion imaging [28].Considering the limitation of fusion imaging, Min et al evaluated the usefulness of SEUS in addition to fusion imaging and demonstrated that $83.3 \%$ (25/30) of small HCCs that are inconspicuous on fusion imaging with B-mode US became conspicuous on fusion imaging with SEUS [29]. Therefore, fusion imaging and SEUS could be regarded as mutually complementary processes to the guidance for biopsy or RFA of focal hepatic lesions. Further studies comparing SEUS guidance and fusion guidance and determining the indications for each guidance tool on biopsy or RFA of focal hepatic lesions could be interesting.

Our study showed a high technical success rate (97.5\%), the results being quite similar to Eso et al [19]. The authors had $92.3 \%$ technical success rate of SEUSguided biopsy for focal hepatic lesions, significantly higher comparing with B-mode US-guided biopsy (76.8\%). This could be explained by the fact that SEUS increased the detection and conspicuity of focal hepatic lesions and visualized the viable area within large necrotic lesions, and thus facilitating adequate targeting and sufficient sampling. Sonazoid might be useful for guidance of focal hepatic lesions in biopsy because of the long duration and high lesion contrast of the Kupffer phase.

Until now there has been no study comparing CEUS using SonoVue and Sonazoid as a guidance tool of hepatic biopsy. SonoVue has been introduced earlier and approved in more countries than Sonazoid [26]. However, Sonazoid is taken up by Kupffer cells, facilitating the persistent enhancement of normal liver parenchyma after vascular phase $[12,13]$. The Kupffer phase begins 10 min after injection and lasts for an hour or more, while vascular phase is limited to 4-6 min [26]. In our study, for four patients with focal hepatic lesions not-detected on B-mode US, we underwent US guided biopsy using the dual split screen mode of B-mode and Kupffer phase image of SEUS. The long duration of the Sonazoid could take more advantage of real-time biopsy or RFA procedure, especially in the case of HCC $[5,6,15,29]$. Furthermore, Sonazoid facilitates diagnosis and grading of $\mathrm{HCC}$ in patients with a high risk for HCC [14,30,31] and a small viable $\mathrm{HCC}$ portion can be depicted using defect-reperfusion imaging by re-injection of Sonazoid [32]. Considering these points, Sonazoid may be a comparable or better guidance tool for biopsy than SonoVue but comparative studies are necessary.

Our study has several limitations. First, even though it was a prospective study, it was only a preliminary study due to the small size of enrolled patients. Therefore, a large scale, multicenter study is needed in order to generalize our hypothesis. Second, on-site image analysis either on B-mode US or on SEUS was evaluated by a single radiologist, and which might, therefore, be somewhat subjective. To overcome this, we strictly defined the grading criteria referring to previous studies $[20,21]$. Third, we included variable-sized hepatic lesions. In large-sized masses it might be easier to perform percutaneous liver biopsy using B-mode US as well as SEUS. Nevertheless, we demonstrated that the technical feasibility for biopsy as well as lesion detection and the conspicuity on SEUS significantly improved compared with that of B-mode US.

In conclusion, SEUS would be helpful for planning the percutaneous biopsy of focal hepatic lesions in terms of the detection, improving lesion conspicuity, tumor viable portion assessment and consequently higher operator confidence, compared with B-mode US.

Acknowledgement: We also thank Bonnie Hami, M.A. (USA) for her editorial assistance in the preparation of this manuscript. This research was partially supported by a research grant from SAMSUNG MEDISON Co., Ltd and by a grant supported by Basic Science Research 
Program through the National Research Foundation of Korea (NRF) funded by the Ministry of Science, ICT \& Future Planning (2017R1A2B4004951).

\section{Conflict of interest: none}

\section{Reference}

1. Cong WM, Wu MC. New insights into molecular diagnostic pathology of primary liver cancer: Advances and challenges. Cancer Lett 2015;368:14-19.

2. Park HJ, Lee MW, Lee MH, et al. Fusion imaging-guided percutaneous biopsy of focal hepatic lesions with poor conspicuity on conventional sonography. J Ultrasound Med 2013;32:1557-1564.

3. Kinkel K, Lu Y, Both M, Warren RS, Thoeni RF. Detection of hepatic metastases from cancers of the gastrointestinal tract by using noninvasive imaging methods (US, CT, MR imaging, PET): a meta-analysis. Radiology 2002;224:748756.

4. Yoon SH, Lee KH, Kim SY, et al. Real-time contrastenhanced ultrasound-guided biopsy of focal hepatic lesions not localised on B-mode ultrasound. Eur Radiol 2010;20:2047-2056.

5. Park HS, Kim YJ, Yu MH, Jung SI, Jeon HJ. Real-time contrast-enhanced sonographically guided biopsy or radiofrequency ablation of focal liver lesions using perflurobutane microbubbles (sonazoid): value of Kupffer-phase imaging. J Ultrasound Med 2015;34:411-421.

6. Kang TW, Lee MW, Song KD, et al. Added Value of Contrast-Enhanced Ultrasound on Biopsies of Focal Hepatic Lesions Invisible on Fusion Imaging Guidance. Korean J Radiol 2017;18:152-161.

7. Claudon M, Cosgrove D, Albrecht T, et al. Guidelines and good clinical practice recommendations for contrast enhanced ultrasound (CEUS) - update 2008. Ultraschall Med 2008;29:28-44.

8. Schlottmann K, Klebl F, Zorger N, Feuerbach S, Scholmerich J. Contrast-enhanced ultrasound allows for interventions of hepatic lesions which are invisible on convential B-mode. Z Gastroenterol 2004;42:303-310.

9. Claudon M, Dietrich CF, Choi BI, et al. Guidelines and good clinical practice recommendations for Contrast Enhanced Ultrasound (CEUS) in the liver - update 2012: A WFUMB-EFSUMB initiative in cooperation with representatives of AFSUMB, AIUM, ASUM, FLAUS and ICUS. Ultrasound Med Biol 2013;39:187-210.

10. Schneider M, Arditi M, Barrau MB, et al. BR1: a new ultrasonographic contrast agent based on sulfur hexafluoridefilled microbubbles. Invest Radiol 1995;30:451-457.

11. Quaia E, Calliada F, Bertolotto M, et al. Characterization of focal liver lesions with contrast-specific US modes and a sulfur hexafluoride-filled microbubble contrast agent: diagnostic performance and confidence. Radiology 2004;232:420-430.

12. Watanabe R, Matsumura M, Chen CJ, Kaneda Y, Ishihara M, Fujimaki M. Gray-scale liver enhancement with Sona- zoid (NC100100), a novel ultrasound contrast agent; detection of hepatic tumors in a rabbit model. Biol Pharm Bull 2003;26:1272-1277.

13. Moriyasu F, Itoh K. Efficacy of perflubutane microbubbleenhanced ultrasound in the characterization and detection of focal liver lesions: phase 3 multicenter clinical trial. AJR Am J Roentgenol 2009; 193:86-95.

14. Tanaka M, Nakashima O, Wada Y, Kage M, Kojiro M. Pathomorphological study of Kupffer cells in hepatocellular carcinoma and hyperplastic nodular lesions in the liver. Hepatology 1996;24:807-812.

15. Minami Y, Kudo M, Hatanaka K, et al. Radiofrequency ablation guided by contrast harmonic sonography using perfluorocarbon microbubbles (Sonazoid) for hepatic malignancies: an initial experience. Liver Int 2010;30:759-764.

16. Meuwly JY, Schnyder P, Gudinchet F, Denys AL. Pulse-inversion harmonic imaging improves lesion conspicuity during US-guided biopsy. J Vasc Interv Radiol 2003;14:335341.

17. $\mathrm{Wu} \mathrm{W}$, Chen $\mathrm{MH}$, Yin SS, et al. The role of contrast-enhanced sonography of focal liver lesions before percutaneous biopsy. AJR Am J Roentgenol 2006;187:752-761.

18. Sparchez Z, Radu P, Zaharia T, et al. Usefulness of contrast enhanced ultrasound guidance in percutaneous biopsies of liver tumors. J Gastrointestin Liver Dis 2011;20:191-196.

19. Eso Y, Takai A, Takeda H, et al. Sonazoid-enhanced ultrasonography guidance improves the quality of pathological diagnosis in the biopsy of focal hepatic lesions. Eur J Gastroenterol Hepatol 2016;28:1462-1467.

20. Rhim H, Choi D, Kim YS, Lim HK, Choe BK. Ultrasonography-guided percutaneous radiofrequency ablation of hepatocellular carcinomas: a feasibility scoring system for planning sonography. Eur J Radiol 2010;75:253-258.

21. Ahn SJ, Lee JM, Lee DH, et al. Real-time US-CT/MR fusion imaging for percutaneous radiofrequency ablation of hepatocellular carcinoma. J Hepatol. 2017;66:347-354.

22. Mishima M, Toh U, Iwakuma N, Takenaka M, Furukawa M, Akagi Y. Evaluation of contrast Sonazoid-enhanced ultrasonography for the detection of hepatic metastases in breast cancer. Breast Cancer 2016;23:231-241.

23. Appelbaum L, Kane RA, Kruskal JB, Romero J, Sosna J. Focal hepatic lesions: US-guided biopsy--lessons from review of cytologic and pathologic examination results. Radiology 2009;250:453-458.

24. Bang N, Bachmann Nielsen M, Vejborg I, Mellon Mogensen A. Clinical report: contrast enhancement of tumor perfusion as a guidance for biopsy. Eur J Ultrasound 2000;12:159-161.

25. Kim PN, Choi D, Rhim H, et al. Planning ultrasound for percutaneous radiofrequency ablation to treat small $(\leq 3$ $\mathrm{cm}$ ) hepatocellular carcinomas detected on computed tomography or magnetic resonance imaging: a multicenter prospective study to assess factors affecting ultrasound visibility. J Vasc Interv Radiol 2012;23:627-634.

26. Claudon M, Dietrich CF, Choi BI, et al. Guidelines and good clinical practice recommendations for contrast enhanced ultrasound (CEUS) in the liver--update 2012: a WFUMB- 
EFSUMB initiative in cooperation with representatives of AFSUMB, AIUM, ASUM, FLAUS and ICUS. Ultraschall Med 2013;34:11-29.

27. Song KD, Lee MW, Rhim H, Cha DI, Chong Y, Lim HK. Fusion imaging-guided radiofrequency ablation for hepatocellular carcinomas not visible on conventional ultrasound. AJR Am J Roentgenol 2013;201:1141-1147.

28. Lee MW, Rhim H, Cha DI, Kim YJ, Lim HK. Planning US for percutaneous radiofrequency ablation of small hepatocellular carcinomas $(1-3 \mathrm{~cm})$ : value of fusion imaging with conventional US and CT/MR images. J Vasc Interv Radiol 2013;24:958-965.

29. Min JH, Lim HK, Lim S, et al. Radiofrequency ablation of very-early-stage hepatocellular carcinoma inconspicuous on fusion imaging with B-mode US: value of fusion imaging with contrast-enhanced US. Clin Mol Hepatol 2014;20:61-70.

30. Korenaga K, Korenaga M, Furukawa M, Yamasaki T, Sakaida I. Usefulness of Sonazoid contrast-enhanced ultrasonography for hepatocellular carcinoma: comparison with pathological diagnosis and superparamagnetic iron oxide magnetic resonance images. J Gastroenterol 2009;44:733741.

31. Arita J, Hasegawa K, Takahashi M, et al. Correlation between contrast-enhanced intraoperative ultrasound using Sonazoid and histologic grade of resected hepatocellular carcinoma. AJR Am J Roentgenol 2011;196:1314-1321.

32. Kudo M. Defect Reperfusion Imaging with Sonazoid ${ }^{\circledR}$ : A Breakthrough in Hepatocellular Carcinoma. Liver Cancer 2016;5:1-7. 